\title{
$\mathrm{AIP} \mid$ chaos \\ An Interdisciplinary Journal of Nonlinear Science
}

\section{Propagation of a solitary fission wave}

A. G. Osborne, G. D. Recktenwald, and M. R. Deinert

Citation: Chaos 22, 023148 (2012); doi: 10.1063/1.4729927

View online: http://dx.doi.org/10.1063/1.4729927

View Table of Contents: http://scitation.aip.org/content/aip/journal/chaos/22/2?ver=pdfcov

Published by the AIP Publishing

\section{Articles you may be interested in}

The ( $G^{\prime} / G$ ) -expansion method for finding traveling wave solutions of nonlinear partial differential equations in mathematical physics

J. Math. Phys. 50, 013502 (2009); 10.1063/1.3033750

Solitary wave propagation in surface stabilized ferroelectric liquid crystal cells

Appl. Phys. Lett. 92, 083510 (2008); 10.1063/1.2841670

A model for the propagation of strain solitary waves in solids with relaxing atomic defects

J. Appl. Phys. 103, 044904 (2008); 10.1063/1.2838478

Generation, propagation, and breaking of internal solitary waves

Chaos 15, 037110 (2005); 10.1063/1.2047907

Mass, momentum, and energy transfer by the propagation of acoustic solitary waves

J. Acoust. Soc. Am. 107, 2398 (2000); 10.1121/1.428626

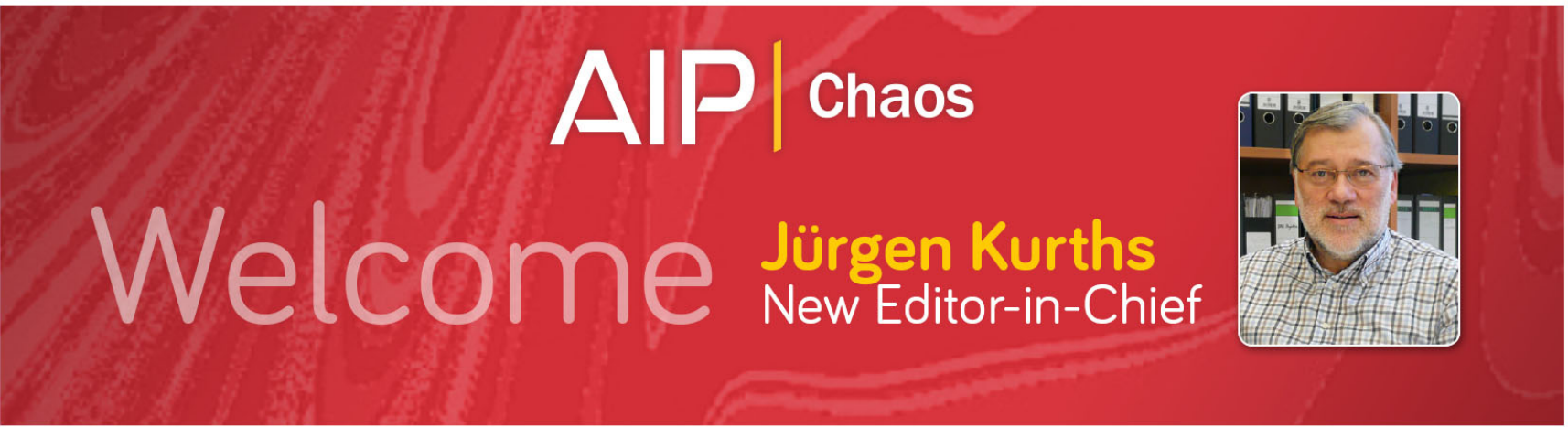




\title{
Propagation of a solitary fission wave
}

\author{
A. G. Osborne, G. D. Recktenwald, and M. R. Deinert ${ }^{\text {a) }}$ \\ Department of Mechanical Engineering, The University of Texas at Austin, Austin, Texas 78715, USA
}

(Received 24 February 2012; accepted 5 June 2012; published online 22 June 2012)

\begin{abstract}
Reaction-diffusion phenomena are encountered in an astonishing array of natural systems. Under the right conditions, self stabilizing reaction waves can arise that will propagate at constant velocity. Numerical studies have shown that fission waves of this type are also possible and that they exhibit soliton like properties. Here, we derive the conditions required for a solitary fission wave to propagate at constant velocity. The results place strict conditions on the shapes of the flux, diffusive, and reactive profiles that would be required for such a phenomenon to persist, and this condition would apply to other reaction diffusion phenomena as well. Numerical simulations are used to confirm the results and show that solitary fission waves fall into a bistable class of reaction diffusion phenomena. (C) 2012 American Institute of Physics. [http://dx.doi.org/10.1063/1.4729927]
\end{abstract}

Reaction diffusion phenomena have been implicated in processes as disparate as fluid flow through soils, neural communication, the mottling of animal coats, traffic flow, and the spread of epidemics. ${ }^{1-5}$ Under the right conditions, reaction and diffusion mechanisms can couple to produce self stabilizing waves that will propagate through the underlying media (e.g., Refs. 6-8). The first description of this phenomenon dates to at least 1844, and a recorded observation of a lone wave traveling unbroken and at constant velocity along a canal. ${ }^{9}$ Edward Teller was the first to present numerical results showing that it could be possible for stable fission waves to form in fertile nuclear materials ${ }^{10}$ and those results have subsequently been confirmed (e.g., Refs. 11-13). Fission waves have recently received wider interest in part because of the involvement of Bill Gates in efforts to design a commercial nuclear reactor based on this phenomena. ${ }^{14}$ Here, we present exact formulations for both the flux profile in a fission wave, and the conditions that are required for a stable wave to form and propagate at a constant velocity. The results are confirmed numerically and show that fission waves fall into a bistable class of solitary wave phenomena.

\section{INTRODUCTION}

A common approach in the study of reaction diffusion waves is to work with the one-dimensional form of the transport equation

$$
\partial_{t} \phi=\partial_{z z} \phi+V \phi
$$

together with the appropriate boundary and initial conditions. For physical applications, the solutions have been found to fall into general classes depending on whether the reaction term, $V$, is greater than zero, less than zero, or oscillatory over the interval of interest. ${ }^{7}$ Solutions for the propagation velocity of reaction-diffusion waves are typically achieved using a

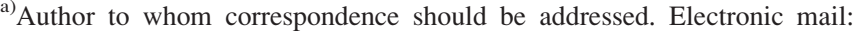
mdeinert@mail.utexas.edu.
}

maxmin approach to Eq. (1) that bounds the velocity ${ }^{7,15}$ based on a knowledge of the reaction and diffusion coefficients.

The non-linear reaction-diffusion equation that underlies fission reactions is only slightly more complicated than Eq. $(1)^{16}$

$$
\alpha \partial_{t} \phi=\partial_{z} D \partial_{z} \phi+V \phi
$$

Here $D$ is the neutron diffusion coefficient and $\alpha$ is the inverse of the neutron velocity. The reaction term $V \phi$ takes into consideration all nuclear reactions as well as neutron leakage. Importantly, $V$ depends on the history of the reaction dynamics and $V \phi$ and $D$ are typically nonlinear. In a one-dimensional system where neutron leakage can be ignored, both $V$ and $D$ are determined by the material composition and if one is known, the other is as well.

While several analytical studies of fission waves have been undertaken, ${ }^{17-20}$ analytical solutions for the flux profiles and the exact requirements for stable wave propagation have not yet been established. In the current contribution, we use a traveling wave coordinate transform to derive the relationship between $D, V$ and $\phi$ that is required for a stable fission wave to persist. The result will hold not only for solitary fission waves but for all constant velocity reaction-diffusion waves in systems governed by equations functionally similar to Eq. (2). We also derive an exact solution for the neutron flux in terms of $\mathrm{D}$ and $\mathrm{V}$. We confirm the results using a numerical implementation of Eq. (2), where $D$ and $V$ are computed based on the evolving isotopics within the simulated system. The simulations also show that fission waves fall into a class of bistable reaction diffusion phenomena.

\section{SOLITARY FISSION WAVES}

In a fission wave fertile material is subjected to a neutron source, the neutrons transmute material downstream and produce fissile material. Eventually, this builds up to a sufficient concentration for criticality, which then produces more neutrons, and so on. Previous 1-D simulations (e.g., Refs. 11, 12, and 21-23) have produced waves that travel slowly, with only a small region being critical at any given time, Fig. 1. 


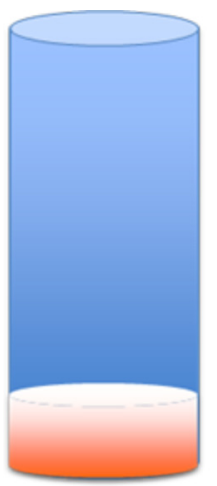

$t_{0}$

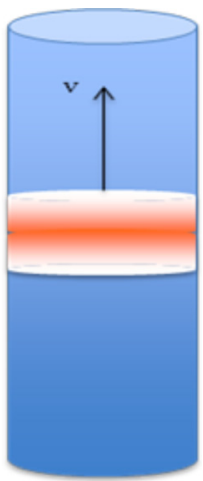

$t>t_{0}$
FIG. 1. Schematic representation of a solitary fission wave moving through fertile material. A fission wave moves through fertile material, breeding fissile material, and then consuming it. Under the proper conditions, the neutron flux profile will move at a constant velocity, $v$.

We use a traveling wave coordinate transformation on Eq. (2), where $\zeta=z-v t$ with $\zeta$ representing a fixed point on the wave

$$
\partial_{\zeta} D \partial_{\zeta} \phi+V \phi=-v \alpha \partial_{\zeta} \phi .
$$

Here, $D$ and $V$ are now understood to be functions of $\zeta$. In order to establish the conditions required for stable propagation of the wave, we follow the approach in Refs. 1-4 to get an ordinary differential equation from Eq. (3). Multiplying through by $d \zeta$ and integrating gives

$$
D \partial_{\zeta} \phi+K(\zeta)=-v \alpha \phi+C
$$

where we define

$$
K(\zeta)=\int V \phi d \zeta
$$

Noting that $K(\zeta)$, and $\phi(\zeta)$ must equal zero at $\zeta=\infty$, we see that $C$ must be 0 as well. The solution to Eq. (4) can be obtained using an integrating factor ${ }^{24}$

$$
\phi=\frac{-\int \frac{K}{D} \exp \left(\int \frac{\alpha v}{D} d \zeta\right) d \zeta}{\exp \left(\int \frac{\alpha v}{D} d \zeta\right)}
$$

It is generally accepted that fission waves could only form in high energy neutron fields and here $\alpha$ would be extremely small and Eq. (6) reduces to

$$
\phi \cong-\int \frac{K}{D} d \zeta .
$$

Rearranging Eq. (4), we get an expression for the front velocity

$$
\mathrm{v}=\frac{q\left(\zeta^{\prime}\right)-K\left(\zeta^{\prime}\right)}{\alpha \phi},
$$

where $q(\zeta)=-D(\zeta d \phi / d \zeta)$.

Equation (8) defines the relationship that must exist between $q(\zeta), K(\zeta)$, and $\phi(\zeta)$ if a constant velocity is to exist across the entire wave profile: the numerator and denominator can only differ by a constant at every location along the flux profile if it is to remain stable.

\section{SIMULATIONS}

Simulations done on fission waves to date have focused on their formation in systems with fast neutron spectra and we take that approach here. We track the concentration of the 15 actinides which dominate the kinetics of fast reactors along with a lumped fission product that captures the behavior of 80 fission product isotopes. The initial material compositions of the system are based on those for a typical sodium cooled fast reactor, ${ }^{25}$ Table I. While Eq. (2) is valid for all neutron energies, we use a one-group (mono-energetic formulation) which is common in fast reactor simulations and has been shown to yield good results for fast reactor kinetics. ${ }^{26}$

The diffusion and reaction terms in Eq. (2) are functions of isotopic concentrations and their corresponding cross sections. The evolution of isotopic concentrations is modeled using a set of coupled first-order transmutation equations, ${ }^{27}$ which are solved using a backward Euler integration scheme. $^{28}$ Accurate one-group reaction cross sections are critical in both cases and are determined as described in the

\begin{tabular}{|c|c|}
\hline Materials tracked & $\begin{array}{c}{ }^{238} \mathrm{U},{ }^{239} \mathrm{~Np},{ }^{238} \mathrm{Pu}{ }^{239} \mathrm{Pu},{ }^{240} \mathrm{Pu},{ }^{241} \mathrm{Pu},{ }^{242} \mathrm{Pu},{ }^{241} \mathrm{Am},{ }^{242 \mathrm{~m}} \mathrm{Am}, \\
{ }^{243} \mathrm{Am},{ }^{242} \mathrm{Cm},{ }^{243} \mathrm{Cm},{ }^{244} \mathrm{Cm},{ }^{245} \mathrm{Cm},{ }^{246} \mathrm{Cm}, \mathrm{FP}\end{array}$ \\
\hline Reactor length & $20 \mathrm{~m}$ \\
\hline Reactor radius & $4 \mathrm{~m}$ \\
\hline${ }^{23} \mathrm{Na}$ concentration & 0.0106 atoms/barn-cm \\
\hline${ }^{238} \mathrm{U}$ concentration (Beginning-of-life) & 0.0133 atoms/barn-cm \\
\hline${ }^{56} \mathrm{Fe}$ concentration & 0.0107 atoms/barn-cm \\
\hline${ }^{238} \mathrm{U}$ density & $12 \mathrm{~g} / \mathrm{cm}^{3}$ \\
\hline${ }^{23} \mathrm{Na}$ density & $0.925 \mathrm{~g} / \mathrm{cm}^{3}$ \\
\hline${ }^{56} \mathrm{Fe}$ density & $7.95 \mathrm{~g} / \mathrm{cm}^{3}$ \\
\hline$\phi(0, t)$ & $1 \times 10^{15} \mathrm{n} / \mathrm{cm}^{2} \mathrm{~s}$ \\
\hline Grid spacing & $\Delta \mathrm{z}=0.1 \mathrm{~cm}$ \\
\hline Time step & $\Delta \mathrm{t}=24 \mathrm{~h}$ \\
\hline
\end{tabular}
supplemental information. ${ }^{29}$

The left-hand boundary condition is a constant surface flux that is used to initiate the solitary fission wave and we

TABLE I. Reactor and simulation parameters. Not shown are concentrations of other materials, all are set to zero at beginning-of-life (BOL). ${ }^{56}$ Fe, ${ }^{23}$ Na, and ${ }^{238} \mathrm{U}$ concentrations reflect a $12.5 \%, 43.75 \%$, and $43.75 \%$ ratio by volume. 
assume a vacuum boundary condition on the right-hand end of the reactor, which is a common assumption in nonreflected reactor cores. ${ }^{16}$ Equation (2) is discretized using a fourth-order, implicit finite-difference scheme, ${ }^{28}$ and the resulting system of equations is solved using NewtonRaphson iteration. ${ }^{28}$ During each iteration, the Jacobian matrix of the system is used to compute a trial flux which is then used to calculate trial isotopic concentrations. The flux and isotopics are then used to recalculate both sides of the discretized Eq. (2). This iteration repeats until the discretized Eq. (2) is satisfied to near machine precision. This approach allows the solutions for both the flux, and the material compositions, to converge simultaneously. It can be shown through an analysis of round off and truncation error that this approach allows the diffusive and reactive terms in Eq. (8) to be distinguished to within machine precision.

\section{DISCUSSION AND CONCLUSION}

Equation (8) requires that the numerator and denominator can only differ by a constant at every location along the flux profile if a constant velocity is to exist and remain stable. Figure 2 shows that this condition is in fact met for a solitary fission wave moving through natural uranium. From the simulated $D(\zeta)$ and $K(\zeta)$ profiles, the wave velocity predicted by Eq. (8) differs from the directly simulated velocity by less than $1 \%$. Importantly, while Eq. (8) was derived for a constant velocity fission wave, the solution would hold for solitary waves in any system that could be described using a reaction diffusion formulation such as Eq. (2). Equation (8) is in fact a generalization of the result for the velocity of a propagating front ${ }^{30}$ which, presented in the context of diffusion limited aggregation of bacterial colonies, was arrived at using mean field theory but gives no restrictions for the con-
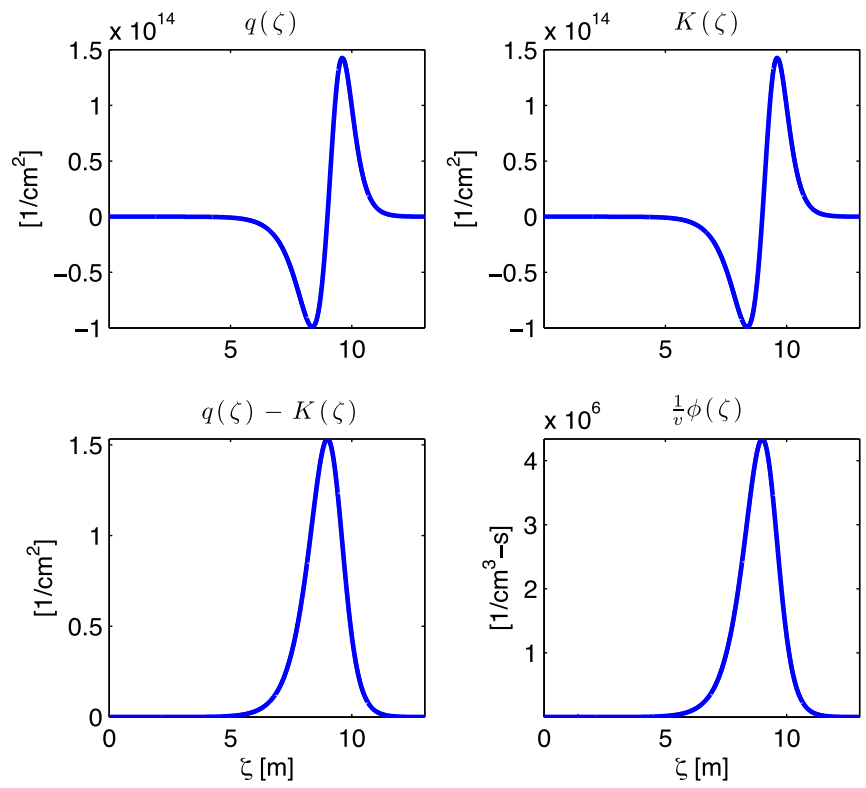

FIG. 2. Diffusive and reactive profiles. Top two plots show the terms in the numerator of Eq. (8), and bottom left shows their difference. Bottom right plot shows the flux profile weighted by the mean neutron velocity. Dividing bottom left by bottom right gives constant wave velocity in $\mathrm{cm} / \mathrm{s}$, and this condition holds at every location. These profiles span the full extent of flux wave.

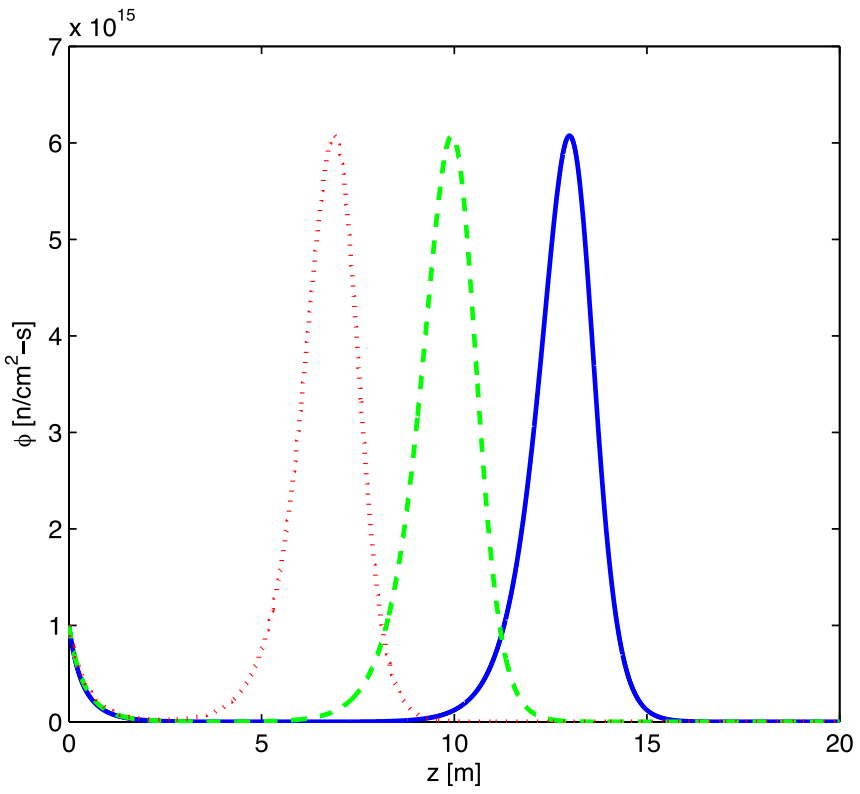

FIG. 3. Flux profiles for 3 time points. Dotted, dashed, and solid lines show fluxes at $\sim 140$ years, 170 years, and 200 years.

ditions required for the entire profile of the wave to propagate at a constant velocity. By contrast, Eq. (8) results from the assumption of a constant velocity and puts strict conditions on the requirements for a that type of wave exist.

In a solitary fission wave, $\phi$ drives the evolution of the isotopics, which then determine the functional forms for $D(\zeta)$, and $V(\zeta)$. Figure 3 shows a flux wave initiated with a constant boundary flux of $1 \times 10^{15} \mathrm{n} / \mathrm{cm}^{2} \mathrm{~s}$ and propagating over a $\sim 200$ year time period. Once the wave is fully initiated, it translates at a constant rate with Eq. (8) giving the conditions required for this to occur. While it is not possible to predict $\phi$ without a simulation to determine $D(\zeta)$ and $K(\zeta)$, Fig. 4 shows a comparison of Eq. (7) to the numerically

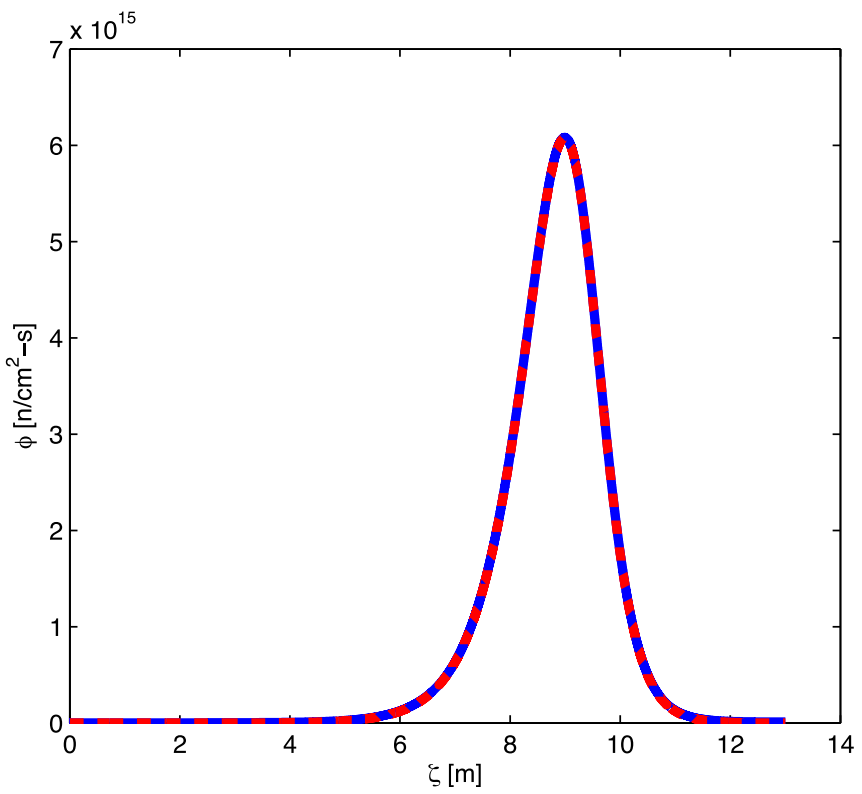

FIG. 4. Neutron flux. The figure shows the computationally determined neutron flux (dashed line) as well as the flux as given by Eq. (7) using the $K(\zeta)$ and $D(\zeta)$ from the simulation results. 


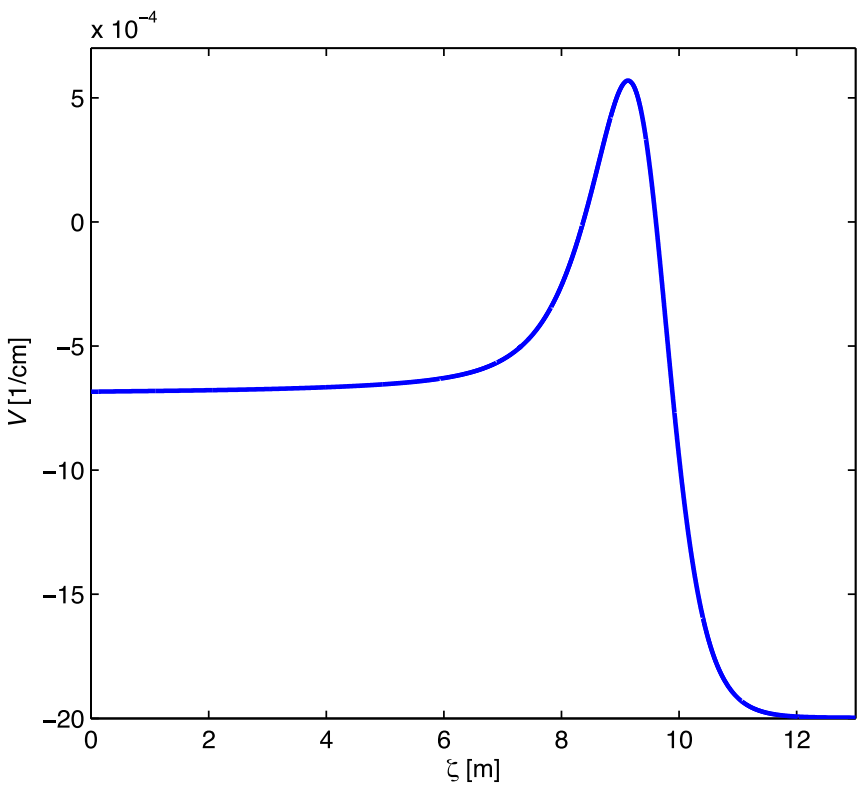

FIG. 5. Spatial dependence of $V$. Neutron flux wave (not shown) peak is centered at $9 \mathrm{~m}$, at $\sim 200$ years. This plot shows that the reaction term changes from positive to negative within the region of interest, which corresponds to the bistable class of reaction-diffusion problems (e.g., Ref. 7).

computed flux when the computationally determined $D(\zeta)$ and $K(\zeta)$ are used. Figure 5 shows that the reaction term in Eq. (2) changes from positive to negative within the domain of interest. This type of potential corresponds to a bistable class of reaction-diffusion problems that have been encountered in other systems (e.g., Ref. 6).

Equation (8) establishes the conditions for stable propagation of a solitary fission wave, and simulations show that these can be met using realistic reactor configurations. While not the subject of this paper, we observe that the intensity of the neutron fields, and the velocity at which the simulated waves move, would result in an extremely high neutron fluence to reactor materials. Whether or not reactors can be built to withstand these conditions will remain to be seen.

\section{ACKNOWLEDGMENTS}

We would like to thank Steve Strogatz for his editorial comments and suggestions and the United States Nuclear Regulatory Commission for supporting this work through grant NRC-38-08-946.

${ }^{1}$ J. Selker, J.-Y. Parlange, and T. Steenhus, Water Resour. Res. 28, 2523 (1992).

${ }^{2}$ P. Liang, Phys. Rev. Lett. 75, 1863 (1995).

${ }^{3}$ J. D. Murray, J. Theor. Biol. 88, 161 (1981).

${ }^{4}$ J. V. Noble, Nature 250, 726 (1974).

${ }^{5}$ P. Zhang and S. C. Wong, Phys. Rev. E 74, 026109 (2006).

${ }^{6}$ R. A. Fisher, Ann. Eugen. 7, 355 (1937).

${ }^{7}$ R. D. Benguria and M. C. Depassier, Phys. Rev. Lett. 77, 1171 (1996).

${ }^{8}$ V. K. Vanag and I. R. Epstein, Phys. Rev. Lett. 88, 088303 (2002).

${ }^{9}$ J. S. Russell, Report of the British Association for the Advancement of Science (Richard and John E. Taylor, London, 1844).

${ }^{10} \mathrm{E}$. Teller, M. Ishikawa, and L. Wood (Lawrence Livermore National Laboratory, 1996).

${ }^{11}$ H. Sekimoto, and K. Ryu, PHYSOR 2000 (American Nuclear Society, Pittsburgh, 2000).

${ }^{12}$ S. P. Fomin et al., Prog. Nucl. Energy 50, 163 (2008).

${ }^{13}$ H. V. Dam, Ann. Nucl. Energy 27, 1505 (2000).

${ }^{14} \mathrm{~T}$. Ellis et al., in International Conference on Advances in Nuclear Power Plants, San Diego, CA, 2010.

${ }^{15}$ A. I. Volpert, V. A. Volpert, and V. A. Volpert, Traveling Wave Solutions of Parabolic Systems (American Mathematical Society, Providence, RI, 1994).

${ }^{16}$ W. M. Stacey, Nuclear Reactor Physics (John Wiley \& Sons, Inc., 2001).

${ }^{17}$ W. Seifritz, Kerntechnik 60, 185 (1995).

${ }^{18}$ W. Seifritz, Kerntechnik 65, 261 (2000).

${ }^{19}$ W. Seifritz, Kerntechnik 67, 130 (2002).

${ }^{20}$ W. Seifritz, Kerntechnik 70, 309 (2005).

${ }^{21}$ H. Sekimoto, K. Ryu, and Y. Yoshimura, Nucl. Sci. Eng. 139, 306 (2001).

${ }^{22}$ S. Fomin et al., Ann. Nucl. Energy 32, 1435 (2005).

${ }^{23}$ S. P. Fomin et al., Probl. At. Sci. Technol. 3, 156 (2007).

${ }^{24} \mathrm{G}$. Birkhoff and G. Rota, Ordinary Differential Equations (Wiley, 1989).

${ }^{25}$ OECD/NEA (OECD, Paris, France, 1995).

${ }^{26}$ L. J. Templin, ANL-5800 (Argonne National Laboratory, Lemont, Illinois, 1963).

${ }^{27}$ H. Bateman, Proc. Cambridge Philos. Soc.: Math. Phys. Sci. 15, 423 (1910).

${ }^{28}$ W. H. Press et al., Numerical Recipes 3rd Edition: The Art of Scientific Computing (Cambridge University Press, 2007).

${ }^{29}$ See supplementary material at http://dx.doi.org/10.1063/1.4729927 for a description of the computational approach methods.

${ }^{30}$ W. Saarloos, Phys. Rep. 386, 29 (2003). 